\title{
The Active Controller Design for AChieving Generalized Projective SYNCHRONIZATION OF HYPERCHAOTIC LÜ AND HyPERCHAOTIC CAI SYSTEMS
}

\author{
Sarasu Pakiriswamy ${ }^{1}$ and Sundarapandian Vaidyanathan ${ }^{1}$ \\ ${ }^{1}$ Department of Computer Science and Engineering \\ Vel Tech Dr. RR \& Dr. SR Technical University \\ Avadi, Chennai-600 062, Tamil Nadu, INDIA \\ sarasujivategmail.com \\ ${ }^{2}$ Research and Development Centre \\ Vel Tech Dr. RR \& Dr. SR Technical University \\ Avadi, Chennai-600 062, Tamil Nadu, INDIA \\ sundarvtu@gmail.com
}

\begin{abstract}
This paper discusses the design of active controllers for achieving generalized projective synchronization (GPS) of identical hyperchaotic Lü systems (Chen, Lu, Lü and Yu, 2006), identical hyperchaotic Cai systems (Wang and Cai, 2009) and non-identical hyperchaotic Lü and hyperchaotic Cai systems. The synchronization results (GPS) for the hyperchaotic systems have been derived using active control method and established using Lyapunov stability theory. Since the Lyapunov exponents are not required for these calculations, the active control method is very effective and convenient for achieving the GPS of the hyperchaotic systems addressed in this paper. Numerical simulations are provided to illustrate the effectiveness of the GPS synchronization results derived in this paper.
\end{abstract}

\section{KEYWORDS}

Active Control, Hyperchaos, Hyperchaotic Systems, Generalized Projective Synchronization, Hyperchaotic Lü System, Hyperchaotic Cai System.

\section{INTRODUCTION}

Chaotic systems are nonlinear dynamical systems which are highly sensitive to initial conditions. This sensitivity of chaotic systems is usually called as the butterfly effect [1]. Small differences in initial conditions (such as those due to rounding errors in numerical computation) yield widely diverging outcomes for chaotic systems.

Hyperchaotic system is usually defined as a chaotic system with more than one positive Lyapunov exponent. The first hyperchaotic system was discovered by O.E. Rössler ([2], 1979).

DOI : 10.5121/ijait.2012.2206 
Since hyperchaotic system has the characteristics of high capacity, high security and high efficiency, it has the potential of broad applications in nonlinear circuits, secure communications, lasers, neural networks, biological systems and so on. Thus, the studies on hyperchaotic systems, viz. control, synchronization and circuit implementation are very challenging problems in the chaos literature [3].

Chaos synchronization problem received great attention in the literature when Pecora and Carroll [4] published their results on chaos synchronization in 1990. From then on, chaos synchronization has been extensively and intensively studied in the last three decades [4-37]. Chaos theory has been explored in a variety of fields including physical systems [5], chemical systems [6], ecological systems [7], secure communications [8-10], etc.

Synchronization of chaotic systems is a phenomenon that may occur when a chaotic oscillator drives another chaotic oscillator. Because of the butterfly effect which causes the exponential divergence of the trajectories of two identical chaotic systems started with nearly the same initial conditions, synchronizing two chaotic systems is seemingly a very challenging problem.

In most of the chaos synchronization approaches, the master-slave or drive-response formalism is used. If a particular chaotic system is called the master or drive system and another chaotic system is called the slave or response system, then the idea of anti-synchronization is to use the output of the master system to control the slave system so that the states of the slave system have the same amplitude but opposite signs as the states of the master system asymptotically. In other words, the sum of the states of the master and slave systems are designed to converge to zero asymptotically, when anti-synchronization appears.

In the recent years, various schemes have been deployed for chaos synchronization such as PC method [4], OGY method [11], active control [12-15], adaptive control [16-20], backstepping design [21-23], sampled-data feedback [24], sliding mode control [25-28], etc.

In generalized projective synchronization (GPS) of chaotic systems [29-30], the chaotic systems can synchronize up to a constant scaling matrix. Complete synchronization [12-13], antisynchronization [31-34], hybrid synchronization [35], projective synchronization [36] and generalized synchronization [37] are particular cases of generalized projective synchronization. GPS has important applications in areas like secure communications and secure data encryption.

In this paper, we deploy active control method so as to derive new results for the generalized projective synchronization (GPS) for identical and different hyperchaotic Lü and hyperchaotic Cai systems. Explicitly, using active nonlinear control and Lyapunov stability theory, we achieve generalized projective synchronization for identical hyperchaotic Lü systems (Chen, Lu, Lü and Yu, [38], 2006), identical hyperchaotic Cai systems (Wang and Cai, [39], 2009) and non-identical hyperchaotic Lü and hyperchaotic Cai systems.

This paper has been organized as follows. In Section 2, we give the problem statement and our methodology. In Section 3, we present a description of the hyperchaotic systems considered in this paper. In Section 4, we derive results for the GPS of two identical hyperchaotic Lü systems. In Section 5, we derive results for the GPS of two identical hyperchaotic Cai systems. In Section 6 , we discuss the GPS of non-identical hyperchaotic Lü and hyperchaotic Cai systems. In Section 7 , we summarize the main results derived in this paper. 


\section{Problem Statement and Our Methodology}

Consider the chaotic system described by the dynamics

$$
\dot{x}=A x+f(x)
$$

where $x \in R^{n}$ is the state of the system, $A$ is the $n \times n$ matrix of the system parameters and $f: R^{n} \rightarrow R^{n}$ is the nonlinear part of the system. We consider the system (1) as the master or drive system.

As the slave or response system, we consider the chaotic system described by the dynamics

$$
\dot{y}=B y+g(y)+u
$$

where $y \in R^{n}$ is the state of the system, $B$ is the $n \times n$ matrix of the system parameters, $g: R^{n} \rightarrow R^{n}$ is the nonlinear part of the system and $u \in R^{n}$ is the controller of the slave system.

If $A=B$ and $f=g$, then $x$ and $y$ are the states of two identical chaotic systems. If $A \neq B$ or $f \neq g$, then $x$ and $y$ are the states of two different chaotic systems.

In the active control approach, we design a feedback controller $u$, which achieves the generalized projective synchronization (GPS) between the states of the master system (1) and the slave system (2) for all initial conditions $x(0), z(0) \in R^{n}$.

For the GPS of the systems (1) and (2), the synchronization error is defined as

$$
e=y-M x
$$

where

$$
M=\left[\begin{array}{cccc}
\alpha_{1} & 0 & \cdots & 0 \\
0 & \alpha_{2} & \cdots & 0 \\
\vdots & \vdots & \ddots & \vdots \\
0 & 0 & \cdots & \alpha_{n}
\end{array}\right]
$$

In other words, we have

$$
e_{i}=y_{i}-\alpha_{i} x_{i}, \quad(i=1,2, \ldots, n)
$$

From (1)-(3), the error dynamics is easily obtained as

$$
\dot{e}=B y-M A x+g(y)-M f(x)+u
$$

The aim of GPS is to find a feedback controller $u$ so that

$$
\lim _{t \rightarrow \infty}\|e(t)\|=0 \text { for all } e(0) \in R^{n} .
$$


Thus, the problem of generalized projective synchronization (GPS) between the master system (1) and slave system (2) can be translated into a problem of how to realize the asymptotic stabilization of the system (6). So, the objective is to design an active controller $u$ for stabilizing the error dynamical system (6) at the origin.

We take as a candidate Lyapunov function

$$
V(e)=e^{T} P e,
$$

where $P$ is a positive definite matrix.

Note that $V: R^{n} \rightarrow R$ is a positive definite function by construction.

We assume that the parameters of the master and slave system are known and that the states of both systems (1) and (2) are measurable.

If we find a feedback controller $u$ so that

$$
\dot{V}(e)=-e^{T} Q e,
$$

where $Q$ is a positive definite matrix, then $\dot{V}: R^{n} \rightarrow R$ is a negative definite function.

Thus, by Lyapunov stability theory [40], the error dynamics (6) is globally exponentially stable and hence the condition (7) will be satisfied. Hence, GPS is achieved between the states of the master system (1) and the slave system (2).

\section{SYSTEMS DESCRIPTION}

The hyperchaotic Lü system ([38], 2006) is described by the dynamics

$$
\begin{aligned}
& \dot{x}_{1}=a\left(x_{2}-x_{1}\right)+x_{4} \\
& \dot{x}_{2}=c x_{2}+x_{1} x_{3} \\
& \dot{x}_{3}=-b x_{3}+x_{1} x_{2} \\
& \dot{x}_{4}=d x_{4}+x_{1} x_{3}
\end{aligned}
$$

where $x_{1}, x_{2}, x_{3}, x_{4}$ are the states and $a, b, c, d$ are constant, positive parameters of the system.

The Lü system (10) exhibits a hyperchaotic attractor when the parameter values are taken as

$$
a=36, \quad b=3, c=20 \text { and } d=1.3
$$

Figure 1 depicts the phase portrait of the hyperchaotic Lü system (10).

The hyperchaotic Cai system ([39], 2009) is described by the dynamics

$$
\begin{aligned}
& \dot{x}_{1}=p\left(x_{2}-x_{1}\right) \\
& \dot{x}_{2}=q x_{1}+r x_{2}+x_{4}-x_{1} x_{3} \\
& \dot{x}_{3}=-s x_{3}+x_{2}^{2} \\
& \dot{x}_{4}=-\varepsilon x_{1}
\end{aligned}
$$


where $x_{1}, x_{2}, x_{3}, x_{4}$ are the states and $p, q, r, s, \varepsilon$ are constant, positive parameters of the system. The Cai dynamics (11) exhibits a hyperchaotic attractor when the parameter values are taken as $p=27.5, q=3, \quad r=19.3, \quad s=2.9$ and $\varepsilon=3.3$

Figure 2 depicts the phase portrait of the hyperchaotic Cai system (11).
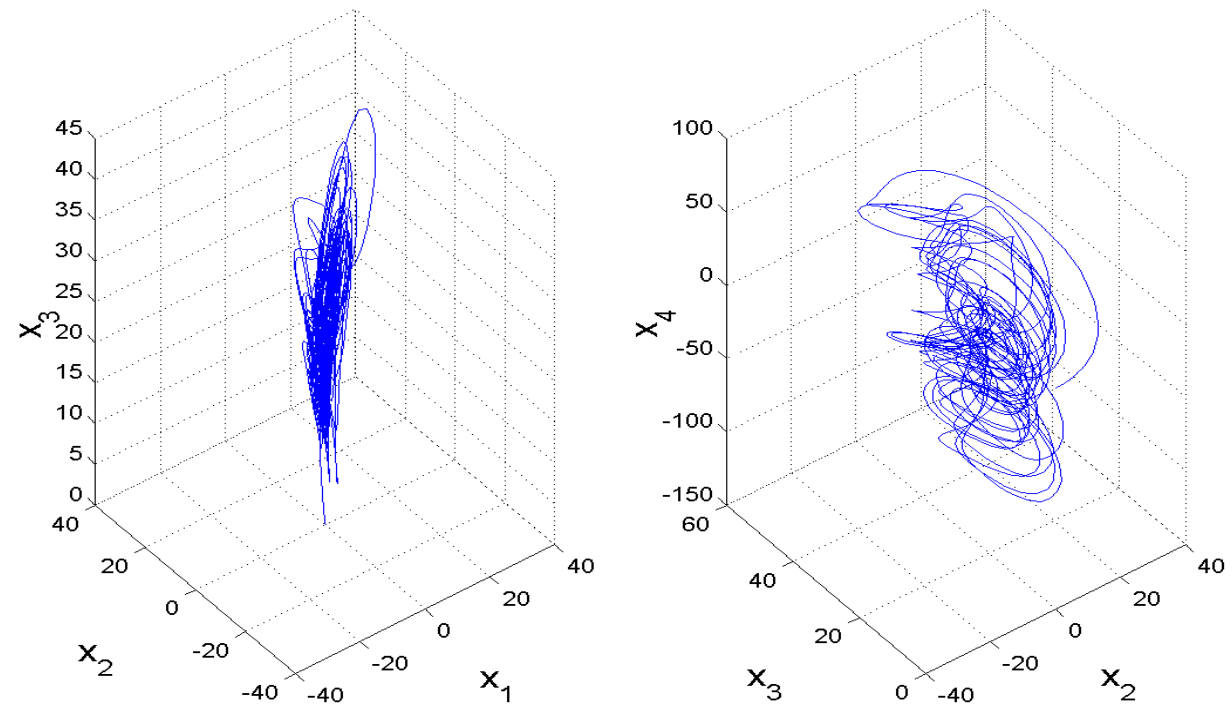

Figure 1. The Phase Portrait of the Hyperchaotic Lü System
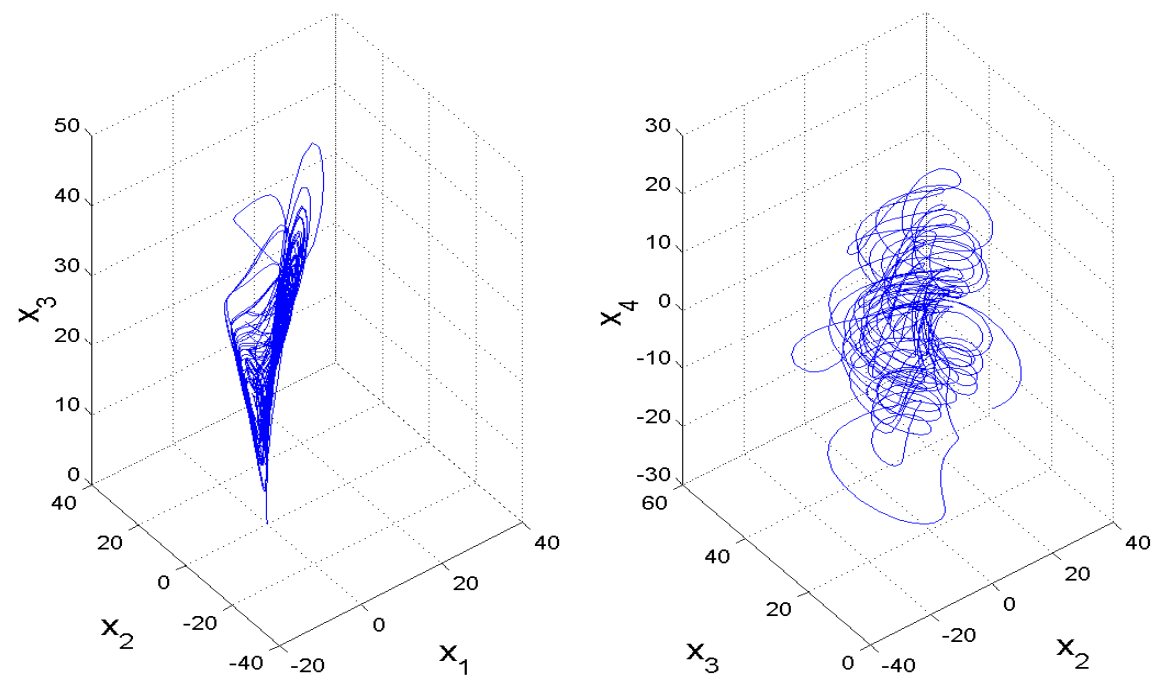

Figure 2. The Phase Portrait of the Hyperchaotic Cai System 


\section{GPS OF IDENTICAL HYPERCHAOTIC LÜ SYSTEMS}

\subsection{Theoretical Results}

In this section, we apply the active nonlinear control method for the generalized projective synchronization (GPS) of two identical hyperchaotic Lü systems ([38], 2006).

Thus, the master system is described by the hyperchaotic Lü dynamics

$$
\begin{aligned}
& \dot{x}_{1}=a\left(x_{2}-x_{1}\right)+x_{4} \\
& \dot{x}_{2}=c x_{2}-x_{1} x_{3} \\
& \dot{x}_{3}=-b x_{3}+x_{1} x_{2} \\
& \dot{x}_{4}=d x_{4}+x_{1} x_{3}
\end{aligned}
$$

where $x_{1}, x_{2}, x_{3}, x_{4}$ are the states and $a, b, c, d$ are positive, constant parameters of the system. The slave system is described by the controlled hyperchaotic Lü dynamics

$$
\begin{aligned}
& \dot{y}_{1}=a\left(y_{2}-y_{1}\right)+y_{4}+u_{1} \\
& \dot{y}_{2}=c y_{2}-y_{1} y_{3}+u_{2} \\
& \dot{y}_{3}=-b y_{3}+y_{1} y_{2}+u_{3} \\
& \dot{y}_{4}=d y_{4}+y_{1} y_{3}+u_{4}
\end{aligned}
$$

where $y_{1}, y_{2}, y_{3}, y_{4}$ are the states and $u_{1}, u_{2}, u_{3}, u_{4}$ are the active controls to be designed.

For the GPS of the systems (12) and (13), the synchronization error $e$ is defined by

$$
e_{i}=y_{i}-\alpha_{i} x_{i}, \quad(i=1,2,3,4)
$$

where the scales $\alpha_{1}, \alpha_{2}, \alpha_{3}, \alpha_{4}$ are real numbers.

The error dynamics is obtained as

$$
\begin{aligned}
& \dot{e}_{1}=-a e_{1}+a\left(y_{2}-\alpha_{1} x_{2}\right)+y_{4}-\alpha_{1} x_{4}+u_{1} \\
& \dot{e}_{2}=c e_{2}-y_{1} y_{3}+\alpha_{2} x_{1} x_{3}+u_{2} \\
& \dot{e}_{3}=-b e_{3}+y_{1} y_{2}-\alpha_{3} x_{1} x_{2}+u_{3} \\
& \dot{e}_{4}=d e_{4}+y_{1} y_{3}-\alpha_{4} x_{1} x_{3}+u_{4}
\end{aligned}
$$

We choose the nonlinear controller as

$$
\begin{aligned}
& u_{1}=a e_{1}-a\left(y_{2}-\alpha_{1} x_{2}\right)-y_{4}+\alpha_{1} x_{4}-k_{1} e_{1} \\
& u_{2}=-c e_{2}+y_{1} y_{3}-\alpha_{2} x_{1} x_{3}-k_{2} e_{2} \\
& u_{3}=b e_{3}-y_{1} y_{2}+\alpha_{3} x_{1} x_{2}-k_{3} e_{3} \\
& u_{4}=-d e_{4}-y_{1} y_{3}+\alpha_{4} x_{1} x_{3}-k_{4} e_{4}
\end{aligned}
$$

where the gains $k_{1}, k_{2}, k_{3}, k_{4}$ are positive constants. 
Substituting (16) into (15), the error dynamics simplifies to

$$
\begin{aligned}
& \dot{e}_{1}=-k_{1} e_{1} \\
& \dot{e}_{2}=-k_{2} e_{2} \\
& \dot{e}_{3}=-k_{3} e_{3} \\
& \dot{e}_{4}=-k_{4} e_{3}
\end{aligned}
$$

Next, we prove the following result.

Theorem 1. The active feedback controller (16) achieves global chaos generalized projective synchronization (GPS) between the identical hyperchaotic Lü systems (12) and (13).

Proof. We consider the quadratic Lyapunov function defined by

$$
V(e)=\frac{1}{2} e^{T} e=\frac{1}{2}\left(e_{1}^{2}+e_{2}^{2}+e_{3}^{2}+e_{4}^{2}\right)
$$

which is a positive definite function on $R^{4}$.

Differentiating (18) along the trajectories of (17), we get

$$
\dot{V}(e)=-k_{1} e_{1}^{2}-k_{2} e_{2}^{2}-k_{3} e_{3}^{2}-k_{4} e_{4}^{2},
$$

which is a negative definite function on $R^{4}$.

Thus, by Lyapunov stability theory [40], the error dynamics (17) is globally exponentially stable. This completes the proof.

\subsection{Numerical Results}

For the numerical simulations, the fourth-order Runge-Kutta method is used to solve the two systems of differential equations (12) and (13) with the active controller (16).

The parameters of the identical hyperchaotic Lü systems are chosen as

$$
a=36, \quad b=3, c=20, \quad d=1.3
$$

The initial values for the master system (12) are taken as

$$
x_{1}(0)=24, \quad x_{2}(0)=-17, \quad x_{3}(0)=-12, \quad x_{4}(0)=18
$$

The initial values for the slave system (13) are taken as

$$
y_{1}(0)=-11, \quad y_{2}(0)=20, \quad y_{3}(0)=-5, \quad y_{4}(0)=34
$$

The GPS scales are taken as $\alpha_{1}=3.5, \quad \alpha_{2}=-2.9, \quad \alpha_{3}=0.8$, and $\alpha_{4}=-1.4$.

We take the state feedback gains as $k_{i}=5$ for $i=1,2,3,4$. 
International Journal of Advanced Information Technology (IJAIT) Vol. 2, No.2, April 2012

Figure 3 shows the GPS synchronization of the identical hyperchaotic Lü systems. Figure 4 shows the time-history of the GPS errors $e_{1}, e_{2}, e_{3}, e_{4}$ for the identical hyperchaotic Lü systems.
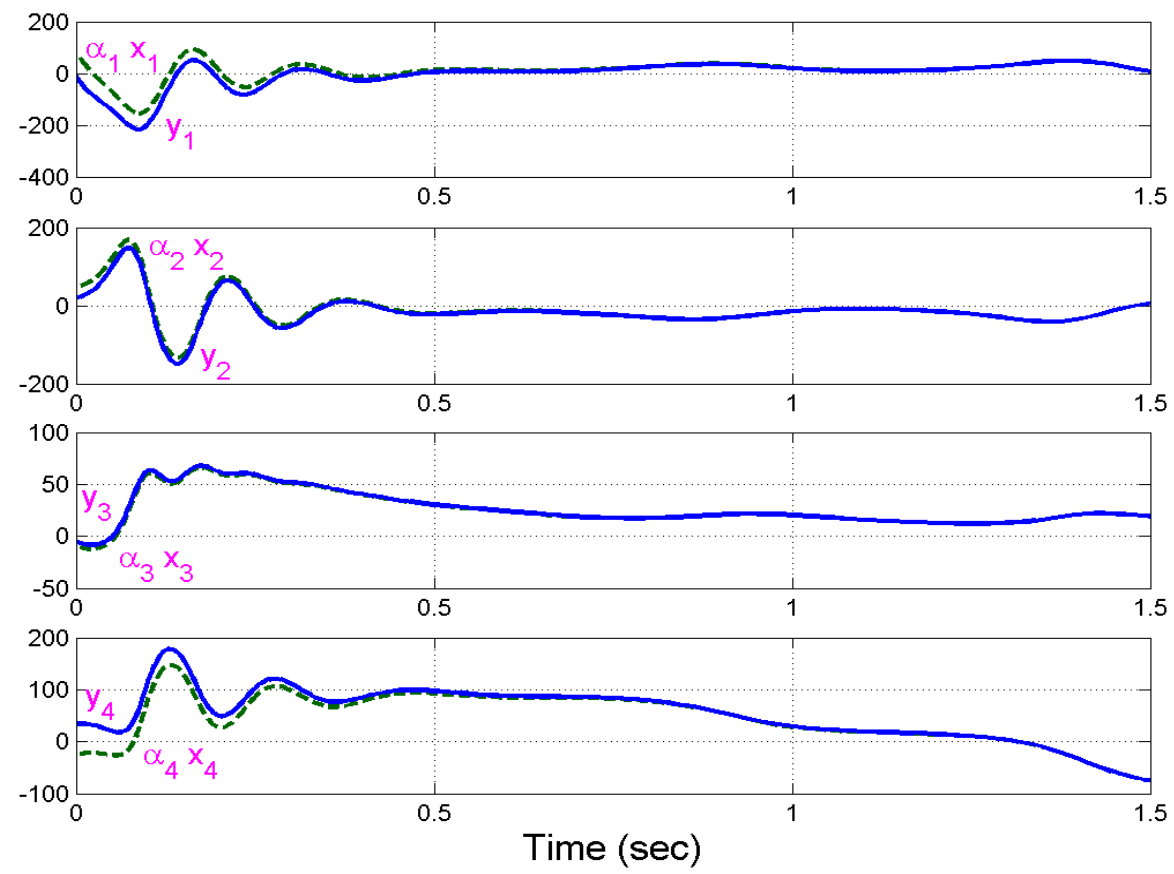

Figure 3. GPS Synchronization of the Identical Hyperchaotic Lü Systems

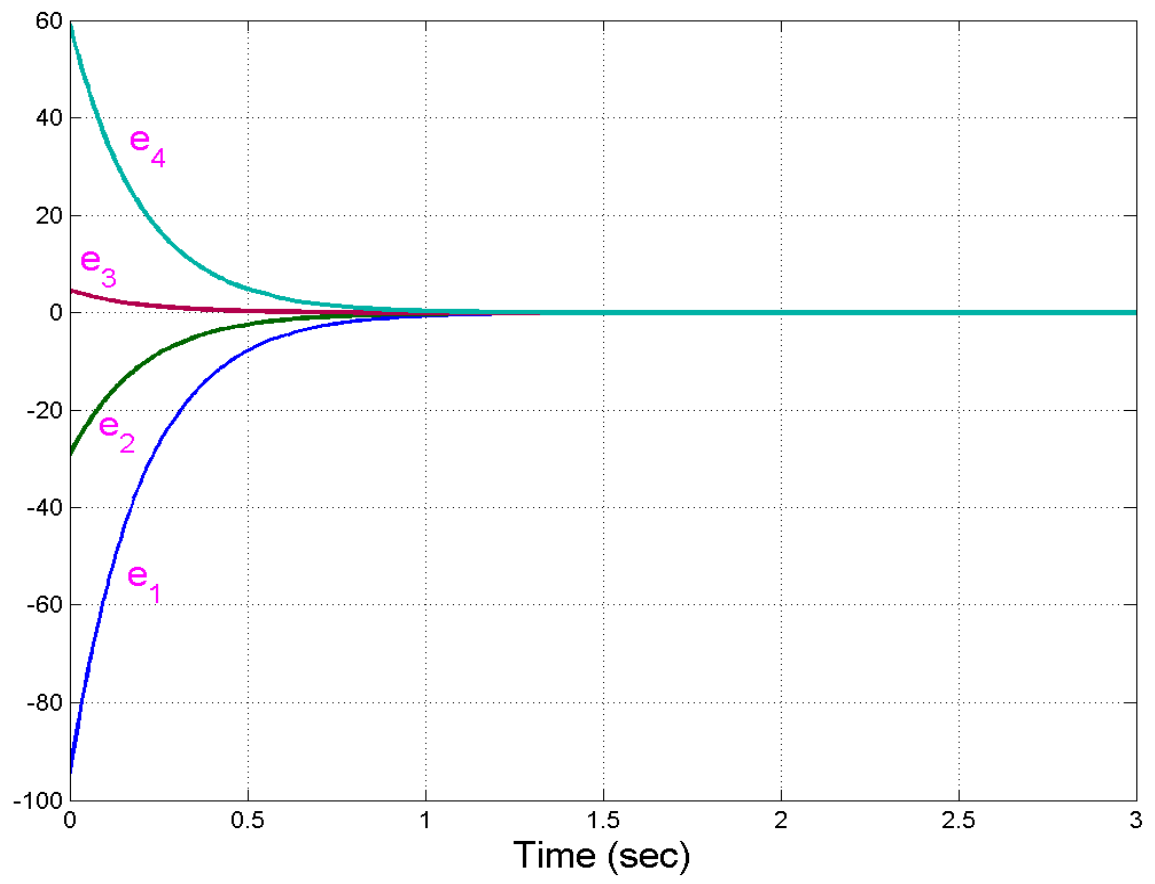

Figure 4. Time History of the GPS Synchronization Error 


\section{GPS OF IdENTICAL HyPERCHAOTIC CAI SySTEMS}

\subsection{Theoretical Results}

In this section, we apply the active nonlinear control method for the generalized projective synchronization (GPS) of two identical hyperchaotic Cai systems ([39], 2009).

Thus, the master system is described by the hyperchaotic Cai dynamics

$$
\begin{aligned}
& \dot{x}_{1}=p\left(x_{2}-x_{1}\right) \\
& \dot{x}_{2}=q x_{1}+r x_{2}+x_{4}-x_{1} x_{3} \\
& \dot{x}_{3}=-s x_{3}+x_{2}^{2} \\
& \dot{x}_{4}=-\varepsilon x_{1}
\end{aligned}
$$

where $x_{1}, x_{2}, x_{3}, x_{4}$ are the states and $p, q, r, s, \varepsilon$ are positive, constant parameters of the system.

The slave system is described by the controlled hyperchaotic Cai dynamics

$$
\begin{aligned}
& \dot{y}_{1}=p\left(y_{2}-y_{1}\right)+u_{1} \\
& \dot{y}_{2}=q y_{1}+r y_{2}+y_{4}-y_{1} y_{3}+u_{2} \\
& \dot{y}_{3}=-s y_{3}+y_{2}^{2}+u_{3} \\
& \dot{y}_{4}=-\varepsilon y_{1}+u_{4}
\end{aligned}
$$

where $y_{1}, y_{2}, y_{3}, y_{4}$ are the states and $u_{1}, u_{2}, u_{3}, u_{4}$ are the active controls to be designed.

For the GPS of the systems (20) and (21), the synchronization error $e$ is defined by

$$
\begin{aligned}
& e_{1}=y_{1}-\alpha_{1} x_{1} \\
& e_{2}=y_{2}-\alpha_{2} x_{2} \\
& e_{3}=y_{3}-\alpha_{3} x_{3} \\
& e_{4}=y_{4}-\alpha_{4} x_{4}
\end{aligned}
$$

where the scales $\alpha_{1}, \alpha_{2}, \alpha_{3}, \alpha_{4}$ are real numbers.

The error dynamics is obtained as

$$
\begin{aligned}
& \dot{e}_{1}=-p e_{1}+p\left(y_{2}-\alpha_{1} x_{2}\right)+u_{1} \\
& \dot{e}_{2}=r e_{2}+q\left(y_{1}-\alpha_{2} x_{1}\right)+y_{4}-\alpha_{2} x_{4}-y_{1} y_{3}+\alpha_{2} x_{1} x_{3}+u_{2} \\
& \dot{e}_{3}=-s e_{3}+y_{2}^{2}-\alpha_{3} x_{2}^{2}+u_{3} \\
& \dot{e}_{4}=-\varepsilon\left(y_{1}-\alpha_{4} x_{1}\right)+u_{4}
\end{aligned}
$$

We choose the nonlinear controller as 


$$
\begin{aligned}
& u_{1}=p e_{1}-p\left(y_{2}-\alpha_{1} x_{2}\right)-k_{1} e_{1} \\
& u_{2}=-r e_{2}-q\left(y_{1}-\alpha_{2} x_{1}\right)-y_{4}+\alpha_{2} x_{4}+y_{1} y_{3}-\alpha_{2} x_{1} x_{3}-k_{2} e_{2} \\
& u_{3}=s e_{3}-y_{2}^{2}+\alpha_{3} x_{2}^{2}-k_{3} e_{3} \\
& u_{4}=\varepsilon\left(y_{1}-\alpha_{4} x_{1}\right)-k_{4} e_{4}
\end{aligned}
$$

where the gains $k_{1}, k_{2}, k_{3}$ are positive constants.

Substituting (24) into (23), the error dynamics simplifies to

$$
\begin{aligned}
& \dot{e}_{1}=-k_{1} e_{1} \\
& \dot{e}_{2}=-k_{2} e_{2} \\
& \dot{e}_{3}=-k_{3} e_{3} \\
& \dot{e}_{4}=-k_{4} e_{4}
\end{aligned}
$$

Next, we prove the following result.

Theorem 2. The active feedback controller (24) achieves global chaos generalized projective synchronization (GPS) between the identical hyperchaotic Cai systems (20) and (21).

Proof. We consider the quadratic Lyapunov function defined by

$$
V(e)=\frac{1}{2} e^{T} e=\frac{1}{2}\left(e_{1}^{2}+e_{2}^{2}+e_{3}^{2}+e_{4}^{2}\right)
$$

which is a positive definite function on $R^{4}$.

Differentiating (26) along the trajectories of (25), we get

$$
\dot{V}(e)=-k_{1} e_{1}^{2}-k_{2} e_{2}^{2}-k_{3} e_{3}^{2}-k_{4} e_{4}^{2},
$$

which is a negative definite function on $R^{4}$.

Thus, by Lyapunov stability theory [40], the error dynamics (25) is globally exponentially stable. This completes the proof.

\subsection{Numerical Results}

For the numerical simulations, the fourth-order Runge-Kutta method is used to solve the two systems of differential equations (20) and (21) with the active controller (24).

The parameters of the identical hyperchaotic Cai systems are chosen as

$$
p=27.5, q=3, \quad r=19.3, \quad s=2.9, \quad \varepsilon=3.3
$$

The initial values for the master system (20) are taken as

$$
x_{1}(0)=15, \quad x_{2}(0)=26, \quad x_{3}(0)=-10, \quad x_{4}(0)=8
$$


The initial values for the slave system (21) are taken as

$$
y_{1}(0)=-21, \quad y_{2}(0)=17, \quad y_{3}(0)=-34, \quad y_{4}(0)=12
$$

The GPS scales are taken as

$$
\alpha_{1}=-1.8, \quad \alpha_{2}=0.6, \quad \alpha_{3}=-3.8, \quad \alpha_{4}=2.7
$$

We take the state feedback gains as

$$
k_{i}=5 \text { for } i=1,2,3,4 \text {. }
$$

Figure 5 shows the GPS synchronization of the identical hyperchaotic Cai systems.

Figure 6 shows the time-history of the GPS synchronization errors for the identical hyperchaotic Cai systems.
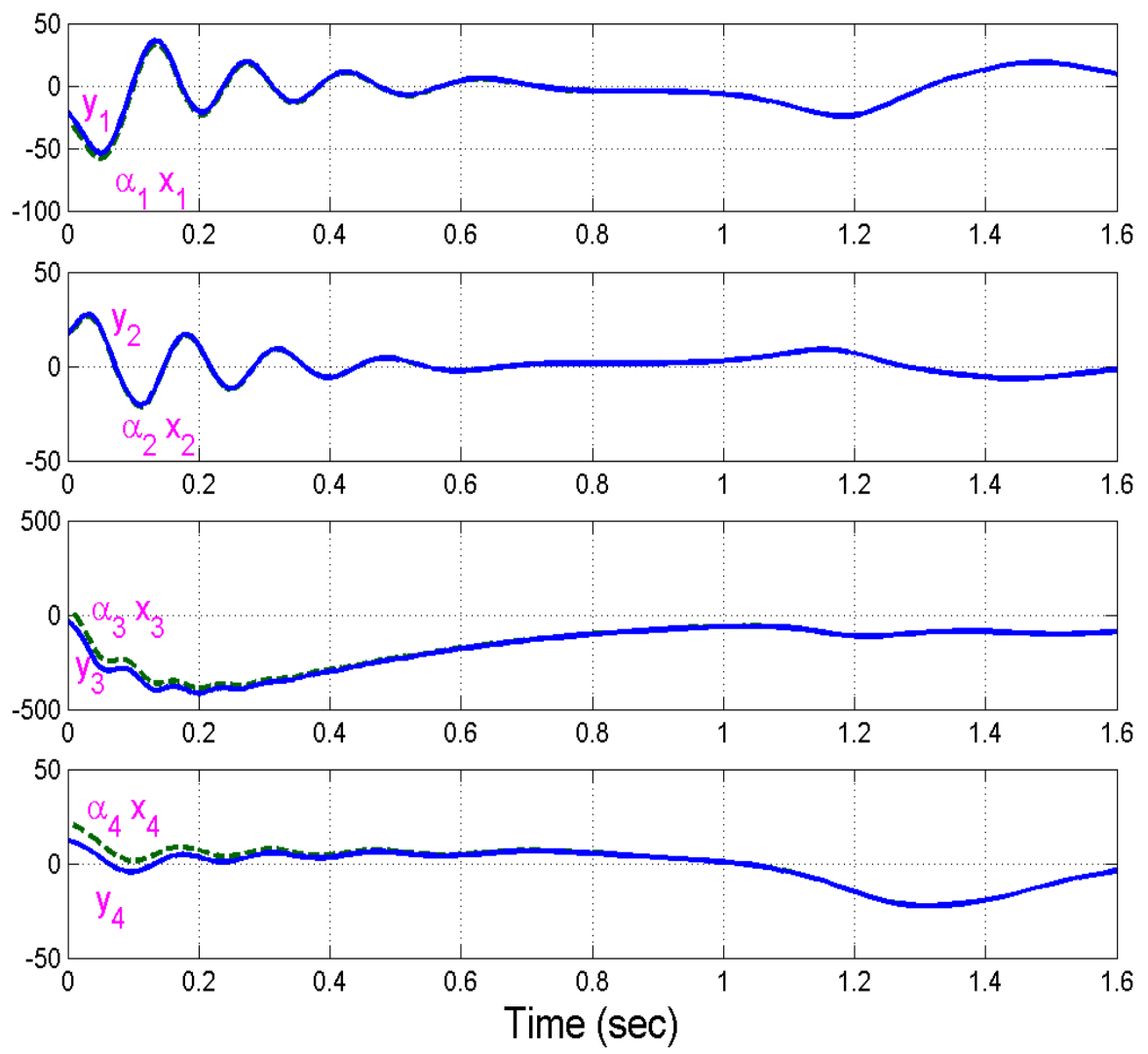

Figure 5. GPS Synchronization of the Identical Hyperchaotic Cai Systems 


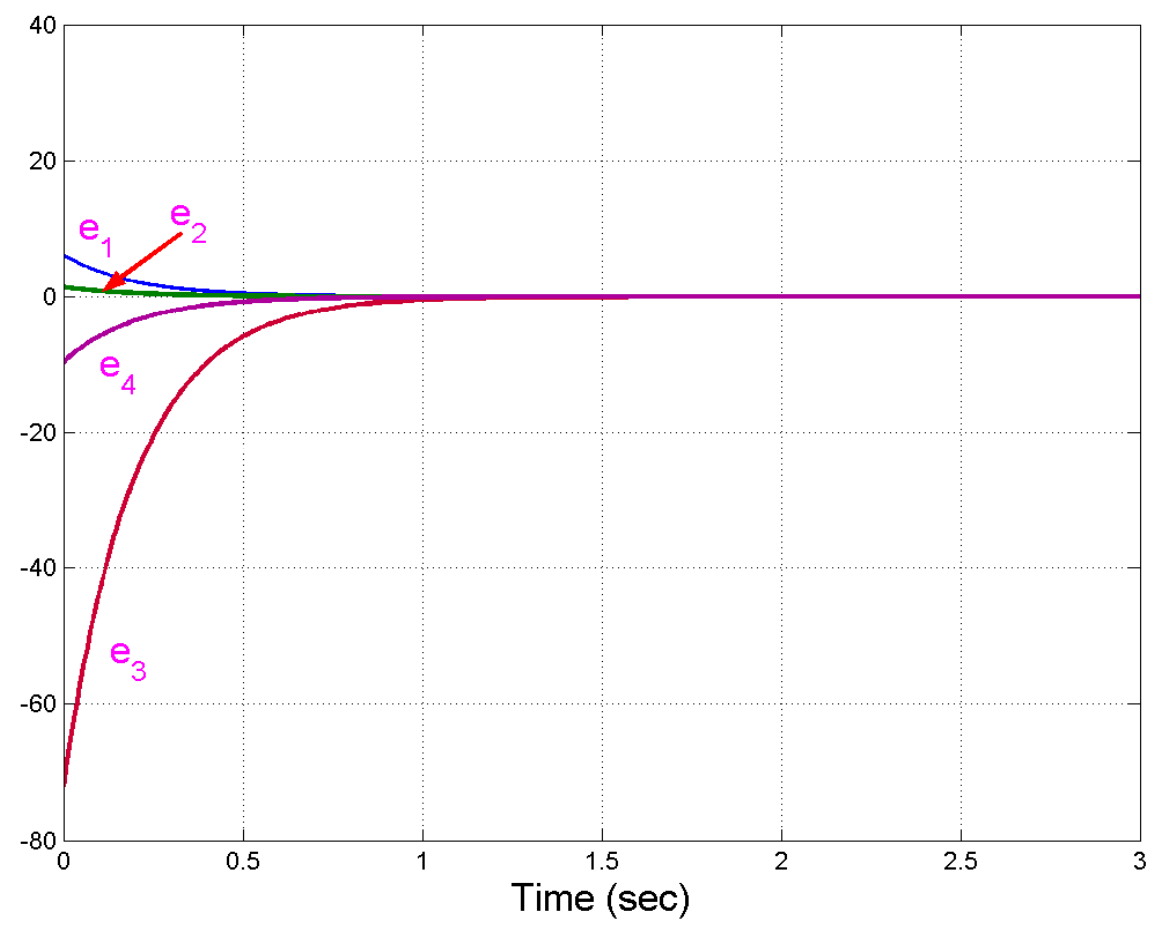

Figure 6. Time History of the GPS Synchronization Error

\section{GPS OF HyPERCHAOTIC LÜ AND HYPERCHAOTIC CAI SYSTEMS}

\subsection{Theoretical Results}

In this section, we apply the active nonlinear control method for the generalized projective synchronization (GPS) of hyperchaotic Lü and hyperchaotic Cai systems.

Thus, the master system is described by the hyperchaotic Lü dynamics

$$
\begin{aligned}
& \dot{x}_{1}=a\left(x_{2}-x_{1}\right)+x_{4} \\
& \dot{x}_{2}=c x_{2}-x_{1} x_{3} \\
& \dot{x}_{3}=-b x_{3}+x_{1} x_{2} \\
& \dot{x}_{4}=d x_{4}+x_{1} x_{3}
\end{aligned}
$$

where $x_{1}, x_{2}, x_{3}, x_{4}$ are the states and $a, b, c, d$ are constant, positive parameters of the system. The slave system is described by the controlled hyperchaotic Cai dynamics

$$
\begin{aligned}
& \dot{y}_{1}=p\left(y_{2}-y_{1}\right)+u_{1} \\
& \dot{y}_{2}=q y_{1}+r y_{2}+y_{4}-y_{1} y_{3}+u_{2} \\
& \dot{y}_{3}=-s y_{3}+y_{2}^{2}+u_{3} \\
& \dot{y}_{4}=-\varepsilon y_{1}+u_{4}
\end{aligned}
$$


where $y_{1}, y_{2}, y_{3}, y_{4}$ are the states, $p, q, r, s, \mathcal{E}$ are positive, constant parameters of the system and $u_{1}, u_{2}, u_{3}, u_{4}$ are the active nonlinear controls to be designed.

For the GPS of the systems (28) and (29), the synchronization error $e$ is defined by

$$
e_{i}=y_{i}-\alpha_{i} x_{i}, \quad(i=1,2,3,4)
$$

where the scales $\alpha_{1}, \alpha_{2}, \alpha_{3}, \alpha_{4}$ are real numbers.

The error dynamics is obtained as

$$
\begin{aligned}
& \dot{e}_{1}=p\left(y_{2}-y_{1}\right)-\alpha_{1}\left[a\left(x_{2}-x_{1}\right)+x_{4}\right]+u_{1} \\
& \dot{e}_{2}=q y_{1}+r y_{2}+y_{4}-y_{1} y_{3}-\alpha_{2}\left[c x_{2}-x_{1} x_{3}\right]+u_{2} \\
& \dot{e}_{3}=-s y_{3}+y_{2}^{2}-\alpha_{3}\left[-b x_{3}+x_{1} x_{2}\right]+u_{3} \\
& \dot{e}_{4}=-\varepsilon y_{1}-\alpha_{4}\left[d x_{4}+x_{1} x_{3}\right]+u_{4}
\end{aligned}
$$

We choose the nonlinear controller as

$$
\begin{aligned}
& u_{1}=-p\left(y_{2}-y_{1}\right)+\alpha_{1}\left[a\left(x_{2}-x_{1}\right)+x_{4}\right]-k_{1} e_{1} \\
& u_{2}=-q y_{1}-r y_{2}-y_{4}+y_{1} y_{3}+\alpha_{2}\left[c x_{2}-x_{1} x_{3}\right]-k_{2} e_{2} \\
& u_{3}=s y_{3}-y_{2}^{2}+\alpha_{3}\left[-b x_{3}+x_{1} x_{2}\right]-k_{3} e_{3} \\
& u_{4}=\varepsilon y_{1}+\alpha_{4}\left[d x_{4}+x_{1} x_{3}\right]-k_{4} e_{4}
\end{aligned}
$$

where the gains $k_{1}, k_{2}, k_{3}, k_{4}$ are positive constants.

Substituting (32) into (31), the error dynamics simplifies to

$$
\begin{aligned}
& \dot{e}_{1}=-k_{1} e_{1} \\
& \dot{e}_{2}=-k_{2} e_{2} \\
& \dot{e}_{3}=-k_{3} e_{3} \\
& \dot{e}_{4}=-k_{4} e_{4}
\end{aligned}
$$

Next, we prove the following result.

Theorem 3. The active feedback controller (32) achieves global chaos generalized projective synchronization (GPS) between the hyperchaotic Lü system (28) and hyperchaotic Cai system (29).

Proof. We consider the quadratic Lyapunov function defined by

$$
V(e)=\frac{1}{2} e^{T} e=\frac{1}{2}\left(e_{1}^{2}+e_{2}^{2}+e_{3}^{2}+e_{4}^{2}\right)
$$

which is a positive definite function on $R^{4}$. 
Differentiating (26) along the trajectories of (33), we get

$$
\dot{V}(e)=-k_{1} e_{1}^{2}-k_{2} e_{2}^{2}-k_{3} e_{3}^{2}-k_{4} e_{4}^{2},
$$

which is a negative definite function on $R^{4}$.

Thus, by Lyapunov stability theory [40], the error dynamics (33) is globally exponentially stable. This completes the proof.

\subsection{Numerical Results}

For the numerical simulations, the fourth-order Runge-Kutta method is used to solve the two systems of differential equations (28) and (29) with the active controller (32).

The parameters of the hyperchaotic Lü system are chosen as

$$
a=36, \quad b=3, c=20, d=1.3
$$

The parameters of the hyperchaotic Cai system are chosen as

$$
p=27.5, q=3, r=19.3, s=2.9, \varepsilon=3.3
$$

The initial values for the master system (28) are taken as

$$
x_{1}(0)=12, \quad x_{2}(0)=24, \quad x_{3}(0)=-39, \quad x_{4}(0)=-17
$$

The initial values for the slave system (29) are taken as

$$
y_{1}(0)=-11, \quad y_{2}(0)=28, \quad y_{3}(0)=7, \quad y_{4}(0)=20
$$

The GPS scales are taken as

$$
\alpha_{1}=2.1, \quad \alpha_{2}=-1.5, \quad \alpha_{3}=-3.6, \quad \alpha_{4}=0.6
$$

We take the state feedback gains as

$$
k_{i}=5 \text { for } i=1,2,3,4
$$

Figure 7 shows the GPS synchronization of the non-identical hyperchaotic Lü and hyperchaotic Cai systems.

Figure 8 shows the time-history of the GPS synchronization errors $e_{1}, e_{2}, e_{3}, e_{4}$ for the nonidentical hyperchaotic Lü and hyperchaotic Cai systems. 

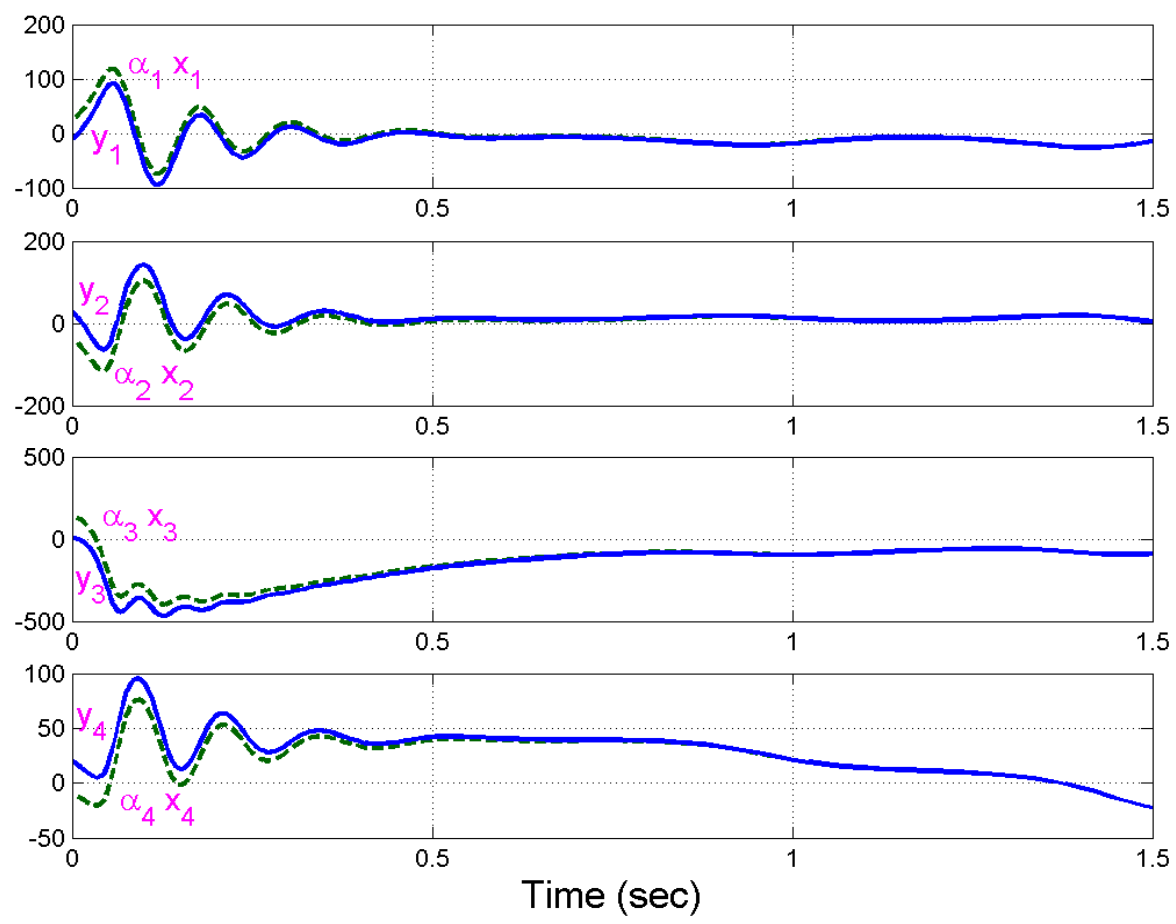

Figure 7. GPS Synchronization of the Hyperchaotic Lü and Hyperchaotic Cai Systems

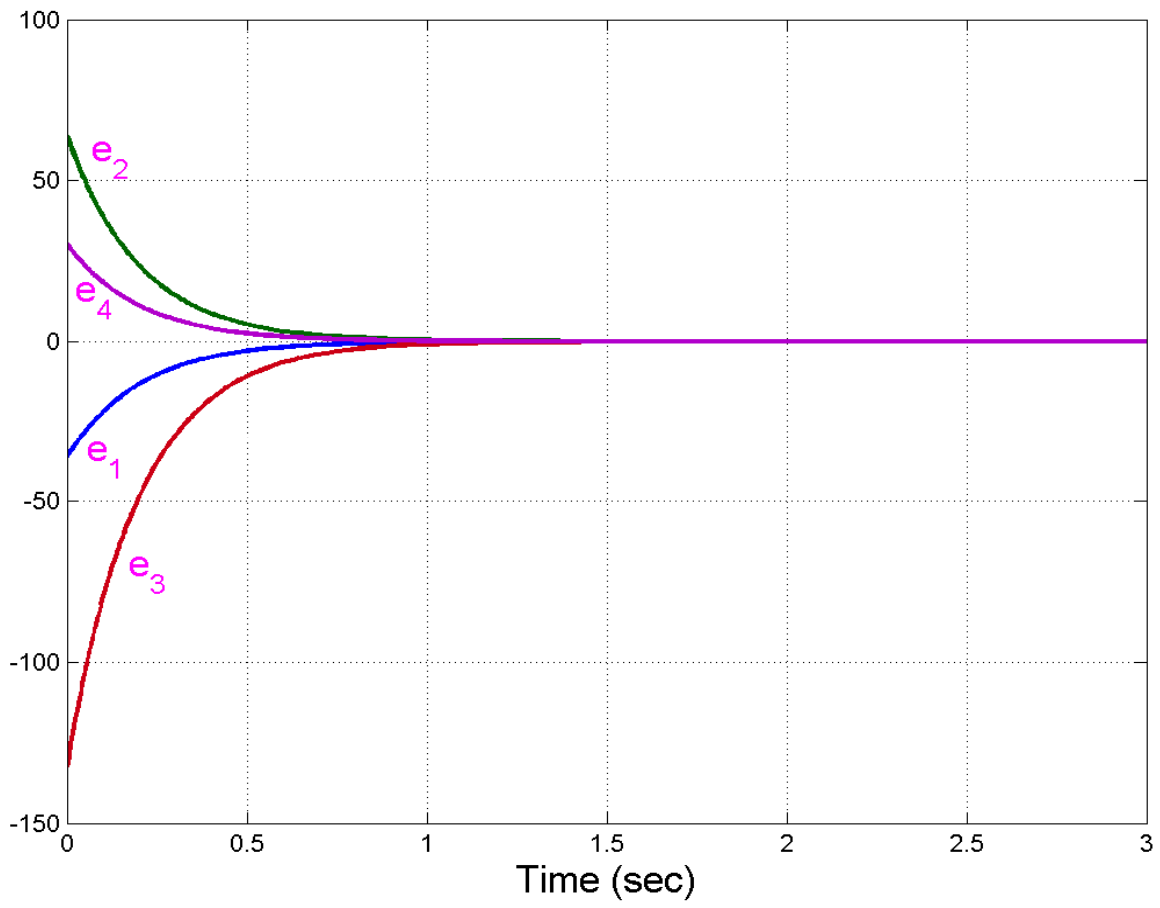

Figure 8. Time History of the GPS Synchronization Error 
International Journal of Advanced Information Technology (IJAIT) Vol. 2, No.2, April 2012

\section{CONCLUSIONS}

In this paper, we had derived active control laws for achieving generalized projective synchronization (GPS) of the following pairs of hyperchaotic systems:

(A) Identical Hyperchaotic Lü Systems (2006)

(B) Identical Hyperchaotic Cai systems (2009)

(C) Non-identical Hyperchaotic Lü and Hyperchaotic Cai systems

The synchronization results (GPS) derived in this paper for the hyperchaotic Lü and hyperchaotic Cai systems have been proved using Lyapunov stability theory. Since Lyapunov exponents are not required for these calculations, the proposed active control method is very effective and suitable for achieving GPS of the hyperchaotic systems addressed in this paper. Numerical simulations are shown to demonstrate the effectiveness of the GPS synchronization results derived in this paper for the hyperchaotic Lü and hyperchaotic Cai systems.

\section{REFERENCES}

[1] Alligood, K.T., Sauer, T. \& Yorke, J.A. (1997) Chaos: An Introduction to Dynamical Systems, Springer, New York.

[2] Rössler, O.E. (1979) “An equation for hyperchaos,” Physics Letters A, Vol. 71, pp 155-157.

[3] Buscarino, A., Fortuna, L. \& Frasca, M. (2009) "Experimental robust synchronization of hyperchaotic circuits," Physica D: Nonlinear Phenomena, Vol. 238, No. 18, pp 1917-1922.

[4] Pecora, L.M. \& Carroll, T.L. (1990) "Synchronization in chaotic systems", Phys. Rev. Lett., Vol. 64, pp 821-824.

[5] Lakshmanan, M. \& Murali, K. (1996) Nonlinear Oscillators: Controlling and Synchronization, World Scientific, Singapore.

[6] Han, S.K., Kerrer, C. \& Kuramoto, Y. (1995) "Dephasing and burstling in coupled neural oscillators", Phys. Rev. Lett., Vol. 75, pp 3190-3193.

[7] Blasius, B., Huppert, A. \& Stone, L. (1999) "Complex dynamics and phase synchronization in spatially extended ecological system", Nature, Vol. 399, pp 354-359.

[8] Feki, M. (2003) "An adaptive chaos synchronization scheme applied to secure communication", Chaos, Solitons and Fractals, Vol. 18, pp 141-148.

[9] Murali, K. \& Lakshmanan, M. (1998) "Secure communication using a compound signal from generalized synchronizable chaotic systems”, Phys. Rev. Lett. A, Vol. 241, pp 303-310.

[10] Yang, T. (2004) "A survey of chaotic secure communication systems," Internat. J. Computational Cognition, Vol. 2, No. 2, pp 81-130.

[11] Ott, E., Grebogi, C. \& Yorke, J.A. (1990) "Controlling chaos”, Phys. Rev. Lett., Vol. 64, pp 11961199.

[12] Ho, M.C. \& Hung, Y.C. (2002) "Synchronization of two different chaotic systems by using generalized active control", Physics Letters A, Vol. 301, pp 424-428.

[13] Chen, H.K. (2005) "Global chaos synchronization of new chaotic systems via nonlinear control", Chaos, Solitons \& Fractals, Vol. 23, pp 1245-1251.

[14] Sundarapandian, V. (2011) "Global chaos synchronization of four-scroll and four-wing chaotic attractors by active nonlinear control," International Journal on Computer Science and Engineering, Vol. 3, No. 5, pp. 2145-2155.

[15] Sundarapandian, V. (2011) "Global chaos synchronization of Li and Liu-Chen-Liu chaotic systems by active nonlinear control," International Journal of Advances in Science and Technology, Vol. 3, No. 1, pp. 1-12.

[16] Liao, T.L. \& Tsai, S.H. (2000) "Adaptive synchronization of chaotic systems and its applications to secure communications", Chaos, Solitons and Fractals, Vol. 11, pp 1387-1396.

[17] Sundarapandian, V. (2011) "Adaptive control and synchronization of hyperchaotic Cai system", International Journal of Control Theory and Computer Modelling, Vol. 1, No. 1, pp 1-13. 
[18] Sundarapandian, V. (2011) "Adaptive synchronization of hyperchaotic Lorenz and hyperchaotic Liu systems”, International Journal of Instrumentation and Control Systems, Vol. 1, No. 1, pp 1-18.

[19] Sundarapandian, V. (2011) "Adaptive control and synchronization of Liu's four-wing chaotic system with cubic nonlinearity," International Journal of Computer Science, Engineering and Applications, Vol. 1, No. 4, pp 127-138.

[20] Sundarapandian, V. \& Karthikeyan, R. (2011) "Global chaos synchronization of Pan and Lü chaotic systems via adaptive control," International Journal of Information Technology, Convergence and Services, Vol. 1, No. 5, pp. 49-66.

[21] Yu, Y.G. \& Zhang, S.C. (2006) "Adaptive backstepping synchronization of uncertain chaotic systems”, Chaos, Solitons and Fractals, Vol. 27, pp 1369-1375.

[22] Wu, X. \& Lü, J. (2003), "Parameter identification and backstepping control of uncertain Lü system," Chaos, Solitons and Fractals, Vol. 18, pp 721-729.

[23] Park, J.H. (2006) "Synchronization of Genesio chaotic system via backstepping approach," Chaos, Solitons and Fractals, Vol. 27, pp 1369-1375.

[24] Yang, T. \& Chua, L.O. (1999) "Control of chaos using sampled-data feedback control”, Internat. J. Bifurcat. Chaos, Vol. 9, pp 215-219.

[25] Sundarapandian, V. (2011) "Global chaos synchronization of four-wing chaotic systems by sliding mode control", International Journal of Control Theory and Computer Modelling, Vol. 1, No. 1, pp 15-31.

[26] Sundarapandian, V. (2011) "Global chaos synchronization of Pehlivan systems by sliding mode control”, International Journal on Computer Science and Engineering, Vol. 3, No. 5, pp 2163-2169.

[27] Sundarapandian, V. (2011) "Sliding mode controller design for the synchronization of ShimizuMorioka chaotic systems", International Journal of Information Sciences and Techniques, Vol. 1, No. 1 , pp 20-29.

[28] Sundarapandian, V. \& Sivaperumal, S. (2011) "Sliding mode controller design for global chaos synchronization of hyperchaotic Lorenz systems," Computer Science and Engineering: An International Journal, Vol. 1, No. 4, pp. 61-71.

[29] Zhou, P., Kuang, F. \& Cheng, Y.M. (2010) "Generalized projective synchronization for fractional order chaotic systems," Chinese Journal of Physics, Vol. 48, no. 1, pp 49-56.

[30] Sarasu, P. \& Sundarapandian, V. (2011) "Active controller design for generalized projective synchronization of four-scroll chaotic systems," International Journal of System Signal Control and Engineering Applications, Vol. 4, No. 2, pp 26-33.

[31] Emadzadeh, A.A. \& Haeri, M. (2005) "Anti-synchronization of two different chaotic systems via active control," World Academy of Science, Engineering and Technology, Vol. 6, pp 62-65.

[32] Sundarapandian, V. \& Karthikeyan, R. (2011) "Anti-synchronization of the hyperchaotic Liu and hyperchaotic Qi systems by active control," International Journal on Computer Science and Engineering, Vol. 3, No. 6, pp 2438-2449.

[33] Sundarapandian, V. \& Karthikeyan, R. (2011) "Anti-synchronization of Pan and Liu chaotic systems by active nonlinear control," International Journal of Engineering Science and Technology, Vol. 3, No. 5, pp 3596-3604.

[34] Sundarapandian, V. (2011) "Anti-synchronization of Lorenz and T chaotic systems by active nonlinear control," International J. Computer Information Systems, Vol. 2, No. 4, pp 6-10.

[35] Sundarapandian, V. (2011) "Hybrid synchronization of hyperchaotic Rössler and hyperchaotic Lorenz systems by active control," International J. Advances in Science and Technology, Vol. 2, No. 4, pp 110.

[36] Mainieri, R. \& Rehacek, J. (1999) "Projective synchronization in three-dimensional chaotic systems," Physical Review Letters, Vol. 82, pp 3042-3045.

[37] Wang, Y.W. \& Guan, Z.H. (2006) "Generalized synchronization of continuous chaotic systems," Chaos, Solitons and Fractals, Vol. 27, pp 97-101.

[38] Chen, A., Lu, J. Lü, J. \& Yu, S. (2006) "Generating hyperchaotic Lü attractor via state feedback control," Physica A, Vol. 364, pp 103-110.

[39] Wang, H. \& Cai, G. (2009) "Controlling hyperchaos in a novel hyperchaotic system," J. Information \& Computing Science, Vol. 4, pp 251-258.

[40] Hahn, W. (1967) The Stability of Motion, Springer, New York. 
International Journal of Advanced Information Technology (IJAIT) Vol. 2, No.2, April 2012

\section{Authors}

Mrs. P. Sarasu obtained her M.E. degree in Embedded System Technologies from Anna University, Tamil Nadu, India in 2007. She obtained her B.E. degree in Computer Science Engineering from Bharatidasan University, Trichy, India in 1998. She is working as an Assistant Professor in the Faculty of Computer Science Engineering, Vel Tech Dr. RR \& Dr. SR Technical University, Chennai. She is currently pursuing her Ph.D degree in Computer Science Engineering from Vel Tech Dr. RR \& Dr. SR Technical University, Chennai. She has published several papers in International Journals and International Conferences. Her current research interests are chaos, control, secure communication and embedded systems.

Dr. V. Sundarapandian obtained his Doctor of Science degree in Electrical and Systems Engineering from Washington University, Saint Louis, USA under the guidance of Late Dr. Christopher I. Byrnes (Dean, School of Engineering and Applied Science) in 1996. He is a Professor in the Research and Development Centre at Vel Tech Dr. RR \& Dr. SR Technical University, Chennai, Tamil Nadu, India. He has published over 240 refereed international publications. He has published over 100 papers in National Conferences and over 60 papers in International Conferences. He is the Editor-in-Chief of International Journal of Mathematics and Scientific Computing, International Journal of Instrumentation and Control Systems, International Journal of

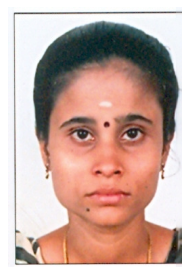
Control Systems and Computer Modelling, etc. His research interests are Linear and Nonlinear Control Systems, Chaos Theory and Control, Soft Computing, Optimal Control, Process Control, Operations Research, Mathematical Modelling and Scientific Computing. He has delivered several Key Note Lectures on Linear and No nlinear Control Systems, Chaos Theory and Control, Scientific Computing using MATLAB/SCILAB, etc. 